\title{
Perfil clínico e assistencial de duas UTIs de um Hospital Universitário através da análise de indicadores de um serviço de fisioterapia
}

\author{
Clinical and care profile of two ICUs in a University Hospital through the analysis of indicators of a \\ physiotherapy service \\ Perfil clínico y asistencial de dos UCI de un Hospital Universitario mediante el análisis de \\ indicadores de un servicio de fisioterapia
}

\author{
Suzanne Guimarães Machado \\ ORCID: https://orcid.org/0000-0002-6519-7233 \\ Universidade Federal de Sergipe, Brasil \\ E-mail: suzanne.machado1@hotmail.com \\ Vanderleia da Silva \\ ORCID: https://orcid.org/0000-0002-3032-3324 \\ Universidade Federal de Sergipe, Brasil \\ E-mail: fisiovanderleia.silva@gmail.com \\ Telma Cristina Fontes Cerqueira \\ ORCID: https://orcid.org/0000-0003-0488-6112 \\ Universidade Federal de Sergipe, Brasil \\ E-mail: telmac@gmail.com \\ Larissa Resende Oliveira \\ ORCID: https://orcid.org/0000-0002-1226-9205 \\ Empresa Brasileira de Serviços Hospitalares, Brasil \\ E-mail: larissa.oliveira.2@ebserh.gov.br \\ Leila Fernanda Oliveira de Jesus Cardoso \\ ORCID: https://orcid.org/0000-0001-7189-0360 \\ Fundação Hospitalar de Saúde, Brasil \\ E-mail: leila.cardoso31@gmail.com \\ Hericalizandra Santa Rosa Santana \\ ORCID: https://orcid.org/0000-0002-3354-2792 \\ Empresa Brasileira de Serviços Hospitalares, Brasil \\ E-mail: hericalizandra@ hotmail.com \\ Juliana de Ávila Barreto \\ ORCID: https://orcid.org/0000-0002-2837-6240 \\ Empresa Brasileira de Serviços Hospitalares, Brasil \\ E-mail: juavilabarreto@gmail.com
}

\begin{abstract}
Resumo
Objetivo: Analisar de forma crítica e comparativa, por meio de indicadores, o perfil clínico e assistencial de pacientes internados nas Unidades de Terapia Intensiva Geral e COVID de um Hospital Universitário de Sergipe. Métodos: Estudo epidemiológico, observacional e longitudinal, desenvolvido no ano de 2020. Foram coletados diariamente, durante o período de internação estabelecido, dados clínicos e assistenciais, a partir dos quais foram compostos os indicadores de qualidade. A análise estatística foi feita através dos testes $t$ de Student e Qui-quadrado, considerando um nível de significância $\mathrm{p}<0,05$. Resultados: A amostra foi composta por 145 pacientes. A principal causa de internação na UTI Covid foi doenças do sistema respiratório, 64.2\%, e na UTI Geral condições cirúrgicas, $42.0 \%$. Taxa de óbito, taxa de uso de VNI, taxa de uso de VMI e taxa de falha de extubação apresentaram diferença estatisticamente significante com $\mathrm{p}<0.05$ quando comparada entre os grupos, sendo todas de valor mais elevado na UTI Covid. Conclusão: Os pacientes internados na unidade Covid obtiveram piores desfechos em parte dos indicadores ventilatórios assim como de mortalidade quando comparados com o grupo UTI Geral. São necessários mais estudos com temas relativos a indicadores, avaliação de qualidade, planejamento e controle dos serviços na área da Fisioterapia.

Palavras-chave: Indicadores de qualidade em assistência a saúde; Cuidados críticos; Serviço hospitalar de fisioterapia; COVID-19.
\end{abstract}

\section{Abstract}

Objective: To analyze critically and comparatively, through indicators, the clinical and care profile of patients admitted to the General Intensive Care Units and COVID of a University Hospital in Sergipe. Methods: 
Epidemiological, observational and longitudinal study, developed in the year 2020. Clinical and care data were collected daily, during the established hospitalization period, from which the quality indicators were composed. A statistical analysis was performed using Student's $t$ and Chi-square tests, considering a level of significance, $\mathrm{p}<0.05$. Results: The sample consisted of 145 patients. The main cause of hospitalization in the ICU Covid was diseases of the respiratory system, $64.2 \%$, and in the General ICU surgical conditions, $42.0 \%$. Death rate, NIV use rate, IMV use rate and extubation failure rate showed statistically significant difference with $\mathrm{p}<0.05$ when compared between groups, all of which are higher in the ICU Covid. Conclusion: Patients admitted to the Covid unit had worse outcomes in part of the ventilatory indicators as well as mortality when compared with the General ICU group. More studies are needed with themes related to indicators, quality assessment, planning and control of services in the field of Physiotherapy.

Keywords: Quality indicators, health care; Critical care; Physical therapy department, hospital; COVID-19.

\section{Resumen}

Objetivo: Analizar crítica y comparativamente, a través de indicadores, el perfil clínico y asistencial de los pacientes ingresados en las Unidades de Cuidados Intensivos Generales y COVID de un Hospital Universitario de Sergipe. Métodos: Estudio epidemiológico, observacional y longitudinal, desarrollado en el año 2020. Se recolectaron datos clínicos y asistenciales diariamente, durante el período de hospitalización establecido, a partir de los cuales se compusieron los indicadores de calidad. El análisis estadístico se realizó mediante pruebas t de Student y chi-cuadrado, considerando un nivel de significancia de $\mathrm{p}<0.05$. Resultados: La muestra estuvo conformada por 145 pacientes. La principal causa de ingreso a la UCI Covid fueron las enfermedades del aparato respiratorio, 64,2\%, ya las condiciones quirúrgicas de la UCI General, 42,0\%. La tasa de mortalidad, la tasa de uso de VNI, la tasa de uso de VMI y la tasa de fracaso de la extubación mostraron diferencias estadísticamente significativas con p <0.05 cuando se compararon entre los grupos, todos los cuales fueron más altos en la UCI Covid. Conclusión: Los pacientes ingresados en la unidad Covid tuvieron peores resultados en parte de los indicadores ventilatorios y de mortalidad en comparación con el grupo de UCI General. Se necesitan más estudios con temas relacionados con indicadores, evaluación de la calidad, planificación y control de los servicios en el campo de la fisioterapia.

Palabras clave: Indicadores de calidad de la atención de salud; Cuidados críticos; servicio hospitalario de fisioterapia; Infecciones por coronavírus.

\section{Introdução}

Existe um interesse crescente por qualidade nos mais diversos setores, sendo algo cada vez mais notável quando se refere à área da saúde, onde o alinhamento de práticas assistenciais, processos e tecnologias utilizadas têm sido consideradas questões prioritárias tanto para o Sistema Único de Saúde (SUS) quanto para a rede privada. Enquanto a gestão se preocupa com a ótima utilização dos seus recursos, já que dentre outros benefícios há diminuição dos custos, os profissionais de saúde preocupam-se com a qualidade da assistência, devido ao compromisso ético intrínseco a esta área, que envolve cuidar do bem mais valioso das pessoas, no caso, a sua saúde (Brasil, 2017).

A qualidade do cuidado em saúde é definida pela Organização Mundial da Saúde (OMS) como "o grau em que os serviços de saúde para indivíduos e populações aumentam a probabilidade de resultados desejados e são consistentes com o conhecimento profissional atual" (Runciman, 2009).

Importante destacar que essa qualidade pode ser influenciada tanto por fatores quantitativos como qualitativos, são exemplos: número de profissionais disponíveis no serviço, características da formação profissional, comportamento do mercado de trabalho, legislação vigente, a estrutura, as políticas e a organização das instituições. Simultaneamente, a avaliação sistemática desses fatores também sofre a influência subjetiva, como a satisfação do usuário (Silva, 2010).

A OMS sugere metas nacionais e internacionais a serem atendidas pelos serviços de saúde, a fim de que se aumente o nível de segurança dos pacientes (Prado, 2015). Dentre esses serviços, se encontram os hospitais, que são responsáveis por complexa parcela da assistência prestada. A inserção de indicadores de segurança nos programas de monitoramento da qualidade representa uma estratégia importante para orientar medidas que promovam/aumentem a segurança do paciente em processo de hospitalização (Gouvêa \& Travassos, 2010). Isso porque conseguem medir de forma quantitativa aspectos do cuidado ao paciente, detectar cuidados abaixo dos padrões referentes à estrutura, processos ou resultado, além de direcionar iniciativas de aperfeiçoamento (Apisarnthanarak, Ajenjo \& Mundy, 2012; Murphy et al., 2012). 
Para O'dwyer, Oliveira e Seta (2009), a dificuldade do monitoramento da qualidade é maior nos casos de serviços de alta complexidade. É nesse contexto que se inserem as Unidades de Terapia Intensiva (UTI), que possuem organização estrutural complexa e de alto custo, o que demanda excelente gerenciamento de seus recursos humanos e materiais. Para isso, uma das intervenções mais importantes é o processo de melhoria do cuidado, que envolve mudanças de comportamento individual e institucional, dinamismo nas rotinas, e necessidade de decisões e atitudes rápidas que, idealmente, utilizem diretrizes baseadas em evidência (Pinto et al., 2014).

Esse processo se torna ainda mais evidente quando se pensa na atual conjuntura da pandemia devido à infecção por Doença do Coronavírus (COVID-19). Pois, embora a maior parte desse perfil de pacientes apresente prognóstico favorável, cerca de $20 \%$ desenvolve formas mais graves, necessitando de internamento hospitalar; e, em torno de 5 a 12\%, necessitam de internação em UTI (Richardson et al., 2020). Assim, um dos principais impactos sobre os serviços de saúde é o rápido aumento na demanda de assistência, principalmente por leitos hospitalares e de UTI. O que torna essencial a existência de um sistema de controle e otimização dos serviços existentes e dimensionamento dos recursos necessários para que se fortaleça a capacidade de resposta dos serviços de saúde frente a esta problemática mundial (Rache et al., 2020).

Embora a maioria dos indicadores de qualidade tenha sido desenvolvida para a área/intervenções médicas, eles são aplicáveis em todos os âmbitos do hospital, e devem englobar todas as profissões que prestem assistência e cuidados à população; o que justifica sua importância para a fisioterapia e prática de reabilitação. Fisioterapeutas podem e devem usar os indicadores como um instrumento de orientação na tomada de decisão clínica, implementação de diretrizes, avaliação da eficácia do tratamento, e relato de conquista de metas (Westyby, Klemm, Li \& Jones, 2015).

Levando em consideração a atuação do profissional fisioterapeuta em uma unidade hospitalar e, especificamente, em UTIs, é importante a realização de estudos e análises que evidenciem a necessidade de um sistema de gestão voltado para a qualidade dos serviços de fisioterapia, assim como para o o uso de ferramentas e indicadores adequados ao alcance dos objetivos da profissão, e que mostrem os resultados do processo de atendimento com foco na segurança do paciente (Prado, 2015).

Os indicadores assistenciais existentes estão ainda pouco elaborados e divulgados; quando existem e formam uma história de coleta de dados, geralmente, ficam restritos dentro das instituições (Cavalheiro \& Eid, 2016). E direcionando para a Fisioterapia, a literatura é extremamente escassa, o que justifica a necessidade de estudos relativos a indicadores, avaliação de qualidade, planejamento e controle dos serviços nessa área. Por isso, esse estudo teve como objetivo analisar de forma crítica e comparativa o perfil clínico e assistencial de pacientes internados nas Unidades de Terapia Intensiva Geral e Covid de um Hospital Universitário através da análise dos indicadores assistenciais do serviço de Fisioterapia.

\section{Metodologia}

\section{Delineamento do Estudo}

Trata-se de um estudo epidemiológico, observacional e longitudinal; aprovado pelo Comitê de Ética em Pesquisa da Universidade Federal de Sergipe (UFS) sob número de parecer 4.086.693 e CAAE 30903520.0.0000.5546, de acordo com a resolução n ${ }^{\circ}$ 466/12 do Conselho Nacional de Saúde. A pesquisa foi desenvolvida no ano de 2020, no Hospital Universitário de Lagarto/SE, que é de médio porte, com perfil assistencial e de porta aberta para atendimento de Urgência e Emergência, focado no âmbito da assistência, ensino, pesquisa e extensão.

\section{Amostra}

A amostra foi constituída de dados clínicos e assistenciais de pacientes admitidos no serviço de Fisioterapia nos setores UTI Covid e UTI Geral do referido hospital, a partir dos quais foram construídos os indicadores, no período de Junho a 
Novembro de 2020. Foram incluídos todos os pacientes internados com idade igual ou superior a 18 anos, independentemente do sexo, causa de internação ou quadro clínico encontrado. Foram excluídos os pacientes que embora tenham passado pelas UTIs no período estabelecido, tenham sido admitidos antes do dia 01 de junho, os que ainda se encontravam internados até o dia 30 de Novembro, e os que foram transferidos para continuidade do tratamento em outra instituição hospitalar, pois dessa forma não tiveram desfecho na internação (alta/óbito).

Os dados foram coletados a partir de análise dos prontuários eletrônicos e registrados em instrumento de coleta próprio através de uma planilha no programa Excel. Foram colhidos para essa pesquisa os seguintes dados/informações: idade; sexo; motivo de internação; comorbidades; datas de admissão hospitalar e na UTI; desfecho (alta/óbito); uso e sucesso ou falha de ventilação não invasiva (VNI); uso e dias de permanência em ventilação mecânica invasiva (VMI); sucesso ou falha nos processos de desmame ventilatório e extubação.

\section{Indicadores de qualidade}

- $\quad$ Taxa de óbito: ( $\mathrm{N}^{\mathrm{o}}$ de óbitos de pacientes internados / $\mathrm{N}^{\circ}$ de pacientes internados x 100)

- $\quad$ Tempo médio de permanência na UTI: ( $\mathrm{N}^{\mathrm{o}}$ da soma de dias que os pacientes permaneceram na UTI / $\mathrm{N}^{\mathrm{o}} \mathrm{de}$ pacientes internados na UTI)

- $\quad$ Taxa de uso de VNI: ( $\mathrm{N}^{\mathrm{o}}$ de pacientes que fizeram uso de VNI / $\mathrm{N}^{\circ}$ de pacientes internados) x 100

- Taxa de sucesso de VNI: ( $\mathrm{N}^{\circ}$ de pacientes com IRpA* não intubados pós VNI/ $\mathrm{N}^{\circ}$ de pacientes com IRpA tratados com VNI) x 100

- $\quad$ Taxa de uso de VMI: ( $\mathrm{N}^{\mathrm{o}}$ de pacientes que fizeram uso de VMI / $\mathrm{N}^{\mathrm{o}}$ de pacientes internados) x 100

- $\quad$ Tempo médio de permanência na VMI: $\left(\mathrm{N}^{\circ}\right.$ da soma de dias que os pacientes fizeram uso de VMI/ $\mathrm{N}^{\circ} \mathrm{de}$ pacientes que fizeram uso de VMI)

- $\quad$ Taxa de sucesso do desmame da VMI: ( $\mathrm{N}^{\mathrm{o}}$ de pacientes que toleraram o TRE*/ $\mathrm{N}^{\mathrm{o}}$ de pacientes submetidos ao TRE x 100)

- $\quad$ Taxa de falha de extubação: (Número de pacientes reintubados em até 48h pós-extubação, incluídos no protocolo de desmame / Número de pacientes que passaram no TRE e foram extubados x 100)

*IRpA: Insuficiência respiratória pulmonar aguda

*TRE: Teste de respiração espontânea

\section{Análise estatística}

Todos os dados foram incluídos em um banco de dados mediante a utilização de planilha eletrônica - Microsoft巴 Office Excel, que posteriormente foi exposto para o programa Statistical Package for Social Sciences for Windows, 11,5 (SPSS Inc, Chicago, IL) onde foram analisados.

A amostra foi composta por dados que seguiam a normalidade/paramétricos, já que a pesquisa contou com um n=145. Uma amostra com pelo menos 30 elementos costuma ser considerada grande o bastante para que a distribuição amostral da média possa ser aproximada por uma normal; o que permite inferir que a distribuição amostral das médias é normal de acordo com o Teorema Central do Limite (Barbetta, 2007).

Foram utilizados os seguintes testes: teste $t$ para amostras independentes a fim de comparar indicadores de perfil clínico e assistencial entre os grupos de pacientes internados nas UTIs Covid e Geral, bem como o teste qui-quadrado para comparação das características entre os grupos para as variáveis categóricas. Para todos os testes foi adotado o nível de significância de $5 \%(\mathrm{p}<0,05)$. 


\section{Resultados}

Inicialmente foi incluído nesse estudo o número de 157 pacientes, sendo todos admitidos pelo serviço de Fisioterapia nas UTIs Covid e Geral no período compreendido entre junho e novembro de 2020. Porém, somente 145 compuseram a amostra final desta pesquisa, sendo 95 da UTI Covid e 50 da UTI Geral, após a exclusão de 12 pacientes que não tiveram desfecho na unidade até o fim da coleta, onde 2 ainda se encontravam internados na UTI e 10 haviam sido transferidos para continuidade de tratamento em outras Unidades Hospitalares, o que impossibilitaria a análise completa desses casos.

A Tabela 1 a seguir mostra dados da caracterização da amostra dos pacientes.

\begin{tabular}{|llll|}
\hline \multicolumn{4}{|c|}{ Tabela 1. Características basais dos participantes. } \\
\hline Variáveis & $\begin{array}{l}\text { UTI Covid } \\
\text { n=95 }\end{array}$ & $\begin{array}{l}\text { UTI Geral } \\
\mathbf{n = 5 0}\end{array}$ & Valor $\boldsymbol{p}$ \\
\hline Sexo, n (\%) & & & \\
Feminino & $45(47.4 \%)$ & $24(48.0 \%)$ & 0.942 \\
Masculino & $50(52.6 \%)$ & $26(52.0 \%)$ & 0.942 \\
Idade (anos), média \pm DP & $64.9 \pm 16.6$ & $64.7 \pm 17.5$ & 0.944 \\
Causa de internação, n (\%) & & & \\
Doenças do sistema respiratório & $61(64.2 \%)$ & $14(28.0 \%)$ & $<0.001^{*}$ \\
Condições cirúrgicas & $5(5.3 \%)$ & $21(42.0 \%)$ & $<0.001^{*}$ \\
Sepse & $6(6.3 \%)$ & $4(8.0 \%)$ & 0.704 \\
Doenças do sistema genitourinário & $6(6.3 \%)$ & $0(0.0 \%)$ & 0.070 \\
Acidente Vascular Cerebral (AVC) & $4(4.2 \%)$ & $3(6.0 \%)$ & 0.633 \\
Doenças oncológicas & $2(2.1 \%)$ & $1(2.0 \%)$ & 0.966 \\
Outros & $11(11.6 \%)$ & $7(14.0 \%)$ & 0.674 \\
Comorbidades, n (\%) & & & \\
Hipertensão arterial sistêmica (HAS) & $45(47.3 \%)$ & $25(50 \%)$ & 0.763 \\
Diabetes Mellitus (DM) & $29(30.5 \%)$ & $18(36.0 \%)$ & 0.503 \\
Tabagismo ou ex-tabagismo & $19(20.0 \%)$ & $7(14.0 \%)$ & 0.371 \\
Obesidade & $15(15.7 \%)$ & $4(8.0 \%)$ & 0.186 \\
Cardiopatia & $11(11.5 \%)$ & $14(28.0 \%)$ & $0.013 *$ \\
Doença neurológica crônica & $16(16.8 \%)$ & $8(16.0 \%)$ & 0.897 \\
Outras & $47(49.4 \%)$ & $17(34.0 \%)$ & 0.074 \\
\hline
\end{tabular}

Dados expostos em número absoluto (n) e percentual (\%), bem como média e desvio padrão (DP). Testes Quiquadrado e Teste t para amostras independentes, ${ }^{*} \mathrm{p}<0,05$. UTI, Unidade de Terapia Intensiva. Fonte: Autores.

Importante observar a homogeneidade existente entre os dois grupos em relação à maioria das variáveis estudadas, exceto no que se refere à causa de internação, onde são expressivos os casos de admissões por doenças do sistema respiratório na UTI Covid e por condições cirúrgicas na UTI Geral, e no tocante à comorbidade prévia cardiopatia, que se apresentou com porcentagem maior no grupo da UTI Geral.

Na Tabela 2 está apresentado o resultado de todos os indicadores elencados nesse estudo. 


\begin{tabular}{|lccc|}
\hline \multicolumn{4}{c|}{ Tabela 2. Resultado dos indicadores clínicos e assistenciais. } \\
\hline Indicadores & $\begin{array}{c}\text { UTI Covid } \\
\mathbf{n = 9 5}\end{array}$ & $\begin{array}{c}\text { UTI Geral } \\
\mathbf{n = 5 0}\end{array}$ & Valor $\boldsymbol{p}$ \\
& $73.6 \%$ & $38.0 \%$ & $<0.001^{*}$ \\
\hline Taxa de óbito & 16 & 15.5 & 0.814 \\
Tempo médio de permanência na UTI (dias) & $40.0 \%$ & $10.0 \%$ & $<0.001^{*}$ \\
Taxa de uso de VNI & $15.78 \%$ & $40.0 \%$ & 0.191 \\
Taxa de sucesso de VNI & $88.4 \%$ & $66.0 \%$ & $0.001^{*}$ \\
Taxa de uso de VMI & 15.8 & 12.4 & 0.185 \\
Tempo médio de permanência na VMI (dias) & $96.4 \%$ & $83.3 \%$ & 0.150 \\
Taxa de sucesso de desmame & $17.8 \%$ & $0.0 \%$ & $0.021^{*}$ \\
Taxa de falha de extubação & & &
\end{tabular}

Dados expostos em número absoluto (n) e percentual (\%). Significância com $* \mathrm{p}<0,05$. Testes Qui-quadrado e Teste t para amostras independentes, *p <0,05. UTI, Unidade de Terapia Intensiva. Fonte: Autores.

Pode-se perceber que taxa de óbito, taxa de uso de VNI, taxa de uso de VMI e taxa de falha de extubação apresentaram diferença estatisticamente significante quando comparada entre os grupos, sendo todas de maior valor na UTI Covid. Notório também, que o tempo médio de permanência dos pacientes nas UTIs e sob uso de VMI foi semelhante. As taxas de sucesso de VNI e de desmame também foram homogêneas entre os grupos.

\section{Discussão}

Neste estudo, foi analisado de forma retrospectiva o perfil clínico e assistencial de pacientes internados nas UTIs Covid e Geral de um Hospital Universitário de Sergipe, a partir de indicadores clínicos e assistenciais do serviço de Fisioterapia das respectivas unidades. Foram inseridos no estudo dados de 145 pacientes internados nos dois setores e observou-se diferença estatisticamente significante nas taxas de óbito, uso de VNI, uso de VMI e falha de extubação.

A taxa nacional de mortalidade esperada para UTIs é de 29\% a 35\% (Ducci \& Padilha, 2004). No que se refere a este estudo, houve diferença significante quando se comparou a variável de desfecho óbito nas unidades, onde a maior parte deles aconteceu na UTI COVID.

As taxas de mortalidade de estudos desenvolvidos nos últimos anos em UTIs Gerais apresentam variações, expressando valores que vão desde 22,58\% (Prece, Cervantes, Mazur \& Visentin, 2016), 38.0\% (Nascimento et al., 2018) e 39.8\% (Barros et al., 2020) até valores mais expressivos como 52.0\% (Castro et al., 2021). A taxa de óbito na UTI Geral pesquisada está de acordo com a literatura, já que o percentual foi de 38\%. Já Batista et al. (2020) analisaram a variação da taxa de letalidade no caso de pacientes com COVID, onde 30,4\% dos seus pacientes em enfermaria vieram a óbito, contra uma proporção de 65,3\% na UTI, valor esse que se aproxima ao obtido nesta pesquisa $(73,6 \%)$.

Interessante ressaltar a relação existente entre desfecho e idade do paciente. Foi observado que nas faixas etárias acima de 60 anos, mais de 50\% dos casos evoluiu para óbito, atingindo valores de $84 \%$ na faixa acima dos 90 anos. Verificouse também que a maioria dos casos apresentou idade entre 50 e 70 anos, corroborando o perfil de pacientes encontrado neste estudo, onde a média de idade foi em torno de 64 anos (Batista et al., 2020). Estudos desenvolvidos em UTIs gerais evidenciam grau de variabilidade quanto a idade dos pacientes, nos quais, assim como o presente estudo, alguns tem a maior parte da amostra composta por idosos (Bastos et al., 2020; Prece, Cervantes, Mazur \& Visentin, 2016; Pauletti, Otaviano, Moraes \& Schneider, 2017), enquanto outros apresentam-se com pacientes de faixa etária menor, com média de idade entre 4044 anos (Barros et al., 2020; Teich, et al. 2020). 
Estudos epidemiológicos também apontam a existência de comorbidades como fator de risco sobre esses desfechos, às quais estariam associadas à maior incidência de sepse e taxa elevada de mortalidade; sendo HAS e DM as comorbidades mais frequentes, assim como os achados do presente estudo (Monteiro, Barros \& Maia, 2016; Moura et al., 2017).

Nesse estudo, a alta taxa de óbitos observada na UTI Covid pode ter sido influenciada pela gravidade dos pacientes internados nesse setor, que frequentemente apresentavam acometimento de múltiplos órgãos, grande número de comorbidades associadas, e que demandavam de terapêuticas como suporte de VMI, uso de drogas vasoativas, necessidade de terapia substitutiva renal, tendo assim prognósticos mais reservados e culminando em maior desfecho de óbito.

Outra questão interessante a ser discutida em se tratando da diferença das taxas de óbito entre as UTIs analisadas é o perfil de pacientes internados em cada uma delas. Na UTI Covid a principal causa de internação foi acometimento do sistema respiratório, sendo em grande parte devido à infecção pela COVID-19, com consequente quadro de insuficiência respiratória e necessidade de suporte ventilatório invasivo. Já na UTI Geral, condições cirúrgicas foram as que levaram a maior parte dos indivíduos a necessitarem de internação. Esse perfil de pacientes frequentemente obtém melhores desfechos clínicos e funcionais, menor tempo de internação e menores taxas de mortalidade.

Estudo realizado por Abelha, Castro, Landeiro, Neves e Santos (2006), avaliou a incidência e os fatores preditivos para mortalidade e tempo de internação dos pacientes admitidos numa UTI cirúrgica, onde encontraram mortalidade geral de 7,6\% durante permanência na UTI e 15,7\% durante internação hospitalar. Logo, os percentuais encontrados de $42.0 \%$ e $5.3 \%$ de pacientes cirúrgicos, nas UTIs Geral e Covid respectivamente, pode ter influenciado na diferença entre o quantitativo de altas nessas Unidades. Esse perfil também pode influenciar no tempo de permanência hospitalar, pois, segundo os autores, em UTI com perfil cirúrgico esta média gira em torno de 4,09 a 10,23 dias, enquanto que em pesquisas que buscaram traçar o perfil de pacientes internados em UTIs gerais o tempo foi de em média 14 dias (Favarin \& Camponogara, 2012; Barros et al., 2020). O que está de acordo com os achados desse estudo, que obteve média de internação de 16 dias na UTI Covid e 15.5 dias na Geral.

É válido explicitar que o hospital onde foi desenvolvida esta pesquisa trata-se de um hospital porta aberta, referência estadual para cirurgia geral e ortopédica dentro da Rede de Atenção às Urgências e Emergências do SUS, e que manteve o fluxo de entrada e realização de cirurgias de urgência/emergência, com limitações para realização de cirurgias eletivas, mesmo nesse período de pandemia. Como não há UTI Cirúrgica na instituição, a UTI Geral é a única responsável por absorver todos os pacientes que necessitam de cuidados intensivos, sejam eles de cunho clínico ou cirúrgico.

As altas taxas de uso de VMI encontradas nos setores estudados, principalmente no grupo COVID, com 88,4\%, precisam ser levadas em consideração quando se pensa em letalidade, já que o uso da VMI por si só é um preditor de mortalidade e está associada a piores desfechos (Amato et al., 2015; Loss et al., 2015). Para Rotta et al. (2018) aproximadamente $33 \%$ dos pacientes admitidos na UTI necessitam de VMI, que tem sido associada a maior mortalidade, maior incidência de pneumonia hospitalar e fraqueza muscular adquirida na UTI, além de custos mais altos, representando $12 \%$ de todos os custos hospitalares.

Em um grande estudo brasileiro com pacientes internados com COVID-19 de todas as regiões do país, os dados apontam que 39\% dos pacientes foram admitidos necessitando de VMI, taxas inferiores às encontradas na UTI Covid no presente estudo, e a taxa de óbito de pacientes com idade entre 20-39 anos que necessitaram de VMI foi de 57\% e para aqueles com 60 anos ou mais foi de 87\% (Ranzani et al., 2021). Evidenciando a relação existente entre uso de VMI, idade avançada e mortalidade. Em outro estudo realizado no México, a mortalidade hospitalar foi de $74 \%$ entre os pacientes ventilados mecanicamente (Ñamendys-Silva, Gutiérrez-Villaseñor \& Romero-González, 2020). 
Ranzani et al. (2021) destacam que as altas taxas de mortalidade em pacientes gravemente enfermos no Brasil, principalmente naqueles que usam VMI, não é uma problemática que surgiu com a pandemia por COVID-19. Mas, provavelmente, o aumento rápido da sobrecarga sobre o sistema de saúde em regiões de poucos recursos agravou essa situação.

O número de dias de utilização de VMI pode evidenciar, mesmo que parcialmente, a qualidade dos cuidados prestados. Pesquisas mostram que 5 a 20\% dos pacientes de UTI utilizam VMI, sendo que 25\% desses por tempo maior que 7 dias (Nassar Junior \& Park, 2014). As pesquisas de Esteban et al. (2002) e Matic et al. (2007) que analisaram pacientes adultos sob uso de ventilação mecânica, apontou tempo médio de permanência em VMI em torno de 7 dias. Estudo prospectivo desenvolvido em UTI Geral adulto obteve média de 9.3 dias, que se assemelha ao obtido no grupo UTI Geral da presente pesquisa, 12.4 dias (Oliveira et al., 2010). Já estudo de coorte retrospectivo com pacientes COVID evidenciou duração média de 29.5 dias (Bastos, 2020), diferentemente dos achados na UTI Covid analisada, onde os valores obtidos foram menores, 15.8 dias.

Houve diferença estatisticamente significativa ao se comparar as taxas de uso de VNI nas unidades, onde este tipo de suporte foi mais utilizado pelo grupo de pacientes Covid. Essa diferença pode ser compreendida pela alta incidência de indivíduos com acometimento do sistema respiratório nesta unidade. Vale ressaltar que foram coletados para essa pesquisa os dados de VNI aplicadas de forma curativa, como estratégia de tratamento de insuficiência respiratória, não sendo contabilizadas aqui as VNIs realizadas de forma profilática, a exemplo de casos pós-extubação ou como terapia de expansão pulmonar, o que pode justificar o baixo número encontrado na UTI Geral.

Estudo com pacientes hipoxêmicos por COVID-19 encontrou taxa de falha de VNI de 88,5\% (Duca et al., 2020). Esses resultados se assemelham aos encontrados nessa pesquisa, onde a taxa de falha de VNI encontrada na UTI Covid foi de $84.22 \%$, o que nos leva a refletir sobre o papel que o suporte ventilatório não invasivo pode ter tido durante essa pandemia; talvez representando bem mais do que a possibilidade de tratar/reverter quadros de insuficiência respiratória, já que poderia, em casos de sucesso, auxiliar na dinâmica organizacional do setor além de evitar procedimentos invasivos como a intubação orotraqueal, proporcionando assim menor uso de recursos com baixa disponibilidade quando se pensava em oferta-demanda nessa situação, a exemplo dos ventiladores mecânicos.

Sobre a baixa taxa de sucesso de VNI em pacientes com COVID, também é importante analisar que, apesar da pneumonia viral causada por esse vírus, em sua forma grave, gerar hipoxemia grave e refratária à oxigenoterapia, com alterações fisiopatológicas que tem certa semelhança às encontradas na Síndrome do Desconforto Respiratório Agudo (SDRA), o subgrupo de pacientes que parece se beneficiar mais da VNI é o que apresenta-se com relação entre pressão parcial de oxigênio no sangue arterial e fração inspirada de oxigênio (PaO2/FiO2) maior que 200 mmHg (Zhan et al., 2012). Estudos anteriores já colocam o diagnóstico de SDRA como um dos fatores independentemente associados à falência de VNI (Adda et al., 2008). Na pandemia, a VNI variou bastante o seu uso de acordo com a localidade, apresentando frequências de uso de $11 \%$ como observado em recente estudo italiano (Grasselli et al., 2020) até 62\% descrito em um estudo desenvolvido na China (Huang et al., 2020).

Embora a Associação Brasileira de Fisioterapia Respiratória (ASSOBRAFIR) não recomende a VNI como estratégia ventilatória de primeira linha para tratamento da COVID, em situações específicas, após avaliação da equipe, ressalta que pode ser feito teste de resposta à VNI por 60 minutos nos casos de paciente com insuficiência respiratória hipoxêmica (Martinez et al., 2021).

As taxas de sucesso de desmame, que representam o quantitativo de pacientes que toleraram o TRE foram satisfatórias nos dois grupos pesquisados. A literatura aponta que o desmame da ventilação mecânica geralmente é bem sucedido, porém em aproximadamente $20 \%$ dos casos pode ocorrer falhas na primeira tentativa do TRE, que é o principal teste diagnóstico para evidenciar se o paciente tem condição de ser extubado com sucesso (Fontela, Eickhoff \& Winkelmann, 2016). 
As principais formas de realizar esse teste são a ventilação mecânica em modo PSV com baixo suporte pressórico (5$7 \mathrm{cmH2O}$ ) e o teste em tubo "T", onde há desconexão do ventilador mecânico e ligação a fonte de suporte de oxigênio (Marinho, Souza, Bahia \& Ferreira, 2020). Porém, no caso de pacientes com COVID não é indicado fazer o teste desconectando-se o paciente do ventilador mecânico, devido ao risco de dispersão de aerossóis e aumento do risco de contaminação, logo nesse perfil o teste deve ser realizado em PSV (Castro, Rocha \& Camillo, 2020).

Estudo nacional que buscou identificar a incidência de falha e sucesso no desmame em UTI, obteve sucesso de desmame em $65 \%$ e falha em $35 \%$ dos casos, ressaltando que consideraram como sucesso o paciente que tolerava a respiração espontânea por um período de no mínimo 48 horas e como fracasso quando o indivíduo retornava à VMI dentro do período de 48 horas (Moreira, Silva \& Bassini, 2011). Esta definição de sucesso/falha, no presente estudo, se enquadra em outro indicador, no caso sucesso/falha de extubação, pois considera o paciente já em respiração espontânea após retirada da via aérea artificial.

Em nossa pesquisa a taxa de falha de extubação foi de $7.8 \%$ na UTI Covid e não houve casos na UTI Geral. A literatura aponta que a ocorrência dessa taxa em UTIs varia entre 5 a 25\%, a depender da população analisada (Reis, Almeida, Silva, Moreira \& Rocha, 2013). Estudo prospectivo em UTI adulto mostrou que 15,2\% da amostra sofreu reintubação, tendo como principais causas: obstrução de vias aéreas superiores pós-extubação (15,6\%); hipoxemia (18,8\%); aumento do trabalho respiratório (40,6\%); redução do nível de consciência (15,6\%); reabordagem cirúrgica $(3,1 \%)$ e outras $(6,3 \%)$ (Oliveira et al., 2010).

Uma questão que pode ser levantada a partir desse comparativo é sobre o tempo que a equipe vem levando para conduzir o processo de extubação desses pacientes, em ambos os setores. Lopes, Jesus, Machado e Reis (2016) trazem em sua pesquisa que o atraso da extubação pode ocorrer em casos de rebaixamento do nível de consciência no momento que os pacientes preenchem os critérios para o desmame; porém já foi visto que pacientes com Escala de Coma de Glasgow $<8$ foram extubados com sucesso, o que mostra que pacientes com nível de consciência rebaixado podem ser desconectados da via aérea artificial logo que sejam considerados aptos, uma vez que tendem a não apresentar melhora da função neurológica com o passar do tempo. O resultado do grupo Covid também pode ter sofrido influência do grande número de pacientes gravíssimos e que evoluíram a óbito ainda em VMI, o que impossibilitou, por vezes, que os mesmos chegassem a fase de desmame da VMI e posterior extubação.

Ter conhecimento do perfil de pacientes e práticas de rotina do serviço influenciam diretamente na qualidade do atendimento prestado por uma UTI, já que a análise proporcionará direcionamento aos gestores e profissionais acerca de decisões a serem tomadas, aquisição de recursos tecnológicos, capacitação dos recursos humanos, assim como reavaliação dos processos de trabalho de forma a possibilitar a adaptação estrutural da unidade às características demográficas e de morbidade da população que ela recebe (Nascimento et al., 2018).

O presente estudo apresenta-se com algumas limitações. Em primeiro lugar, este estudo foi uma análise descritiva que não teve como objetivo investigar relações de interdependência entre as variáveis dependentes e independentes, o que poderia enriquecer a compreensão acerca dos resultados obtidos, assim como a contribuição para adaptações a melhorias do cuidado. Em segundo lugar, as UTIs onde o estudo se desenvolveu não conta com a utilização de nenhum instrumento de classificação de gravidade do doente, a exemplo de escalas como APACHE. Sabe-se que a utilização de escalas como essa em UTIs tem viabilizado melhor avaliação do desempenho das unidades, tendo como objetivo maior a eficiência do tratamento utilizado (Denti \& Pigatto, 2019). 


\section{Conclusão}

Este estudo investigou o perfil clínico e assistencial de pacientes internados nas Unidades de Terapia Intensiva Geral e Covid de um Hospital Universitário. De acordo com nossos resultados pode-se afirmar que a maior parte desse perfil de pacientes foi composta por idosos do sexo masculino, que possuiam mais frequentemente as comorbidades HAS, DM e cardiopatias; teve como causa de internação principal o acometimento do sistema respiratório (UTI Covid) e condições cirúrgicas (UTI Geral). Houve diferença estatisticamente significante entre as UTIs, com valores mais altos na unidade Covid no que se refere as taxas de óbito, uso de VMI, uso de VNI e falha de extubação.

Ao expandir os indicadores assistenciais para além da instituição hospitalar essa pesquisa contribui com a divulgação e elaboração de indicadores de qualidade na área da Fisioterapia, de forma a viabilizar a utilização por mais serviços de saúde, o que pode impactar de forma positiva na segurança do paciente, monitorização da qualidade dos serviços prestados, além de crescimento e fortalecimento da profissão.

Diante disso, é notória a necessidade do desenvolvimento de mais estudos com temas relativos a indicadores, avaliação de qualidade, planejamento e controle dos serviços na área da Fisioterapia. A exemplo de pesquisas que investiguem ferramentas para o gerenciamento de boas práticas na área, ensaios clínicos comparando aplicabilidade e eficácia de técnicas distintas para um mesmo objetivo, assim como a qualidade assistencial em diferentes setores ou instituições.

\section{Referências}

Abelha, J. F., Castro, M. A., Landeiro, N. M., Neves, A. M., \& Santos, C. C. (2006). Mortalidade e tempo de internação em Unidade de Terapia Intensiva Cirúrgica. Revista Brasileira de Anestesiologia, 56(1).

Adda, M., Coquet, I., Darmon, M., Thiery, G., Schlemmer, B., \& Azoulay, E. (2008). Predictors of noninvasive ventilation failure in patients with hematologic malignancy and acute respiratory failure. Critical Care Medicine, 36(10), 2766-2772.

Amato, M. B. P., Meade, M. O., Slutsky, A. S., Brochard, L., Costa, E. L. V., Schoenfeld, D. A., \& Brower, R. G. (2015). Driving pressure and survival in the acute respiratory distress syndrome. New England Journal of Medicine, 372(8), 747-755.

Apisarnthanarak, A., Ajenjo, M. C., \& Mundy, L. (2012). Infection Prevention and Control: A Global Perspective on a Health Care Crisis. In B. M. Soule; Z. A. Memish; P. Malani, Best Practices in Infection Prevention and Control: Na International Perspective. (pp. 1-10). USA: Joint Commission International.

Barbetta, P. A. (2007). Estatística Aplicada às Ciências Sociais. Florianópolis: Editora da UFSC.

Barros, F. L. O. S., Carvalho, K. M. M. B., Falcão, A. R. N., Ribeiro Fº, J. D. M., Gadelha, R. R. M., \& Campos, D. C. O. (2020). Perfil epidemiológico dos pacientes atendidos em uma unidade de terapia intensiva cardiopulmonar de um hospital de referência do Ceará. Revista de Medicina da UFC, 60(2), 26-30.

Bastos, G. A. N., Ajambuja, A. Z., Polanczyk, C. A., Gräf, D. D., Zorzo, I. W., Maccari, J. G., \& Scotta, M. C. (2020). Características clínicas e preditores de ventilação mecânica em pacientes com COVID-19 hospitalizados no sul do país. Revista Brasileira de Terapia Intensiva, $32(4)$, $487-492$.

Batista, A., Antunes, B., Faveret, G., Peres, I., Marchesi, J., Cunha, J. P., \& Bozza, F. (2020). Análise socioeconômica da taxa de letalidade da COVID-19 no Brasil. Núcleo de Operações e Inteligência em Saúde (NOIS)/ PUCRio - Nota técnica.

Brasil. (2017). Agência Nacional de Vigilância Sanitária. Assistência Segura: Uma Reflexão Teórica Aplicada a Prática. Agência Nacional de Vigilância Sanitária. Brasília: Anvisa.

Cavalheiro, L.V., \& Eid, R.C. (2016). Indicadores de qualidade em terapia intensiva. In Martins, J. A.; Andrade, F. M. D. \& Beraldo, M. A., PROFISIO Programa de Atualização em Fisioterapia em Terapia Intensiva Adulto: Ciclo 6. (pp. 127-149). Porto Alegre: Artmed Panamericana (Sistema de Educação Continuada a Distância).

Castro, L. A., Rocha, A. R. M., \& Camillo, C. A. (2020). Desmame da ventilação mecânica em pacientes com COVID-19*. ASSOBRAFIR Ciência, (11), 175182.

Castro, M. L. M., Almeida, F. C. A., Amorim, E. H., Carvalho, A. I. L. C., Costa, C. C., \& Cruz, R. A. O. (2021). Perfil de pacientes de uma unidade de terapia intensiva de adultos de um município paraibano. Revista Electrónica Enfermería Actual en Costa Rica, (40).

Denti, I. A., \& Pigatto, D. F. (2019). Perfil e prognóstico de pacientes internados em Unidade de Terapia Intensiva através da utilização da Escala de Apache II. Revista Perspectiva, 44(165), 123-133.

Duca, A., Memaj, I., Zanardi, F., Preti, C., Alesi, A., Bella, L. D., \& Cosentini, R. (2020). Severity of respiratory failure and outcome of patients needing a ventilatory support in the Emergency Department during Italian novel coronavirus SARSCoV2 outbreak: Preliminary data on the role of Helmet CPAP and Non-Invasive Positive Pressure Ventilation. EClinicalMedicine. 
Ducci, A. J., \& Padilha, K. G. (2004). Caracterização da gravidade de pacientes adultos internados em Unidade de Terapia Intensiva: Análise evolutiva segundo o Therapeutic Intervention Scoring System. Revista Brasileira de Terapia Intensiva.

Esteban, A., Anzueto, A., Frutos, F., Alía, I., Brochard, L., Stewart, T E., ... \& Tobin, M. J. (2002). Characteristics and outcomes in Adults patients receveing mechanical ventilation. Journal of the American Medical Association, 287(3), 345-55.

Favarin, S. S., \& Camponogara, S. (2012). Perfil dos pacientes internados na Unidade de Terapia Intensiva Adulto de um Hospital Universitário. Revista de Enfermagem UFSM, 2(2), 320-329.

Fontela, P. C., Eickhoff, H. M., \& Winkelmann, E. R. (2016). Incidência e fatores associados ao desmame. Ciência \& Saúde, 9(3), 167-173.

Gouvêa, C. S. D., \& Travassos, C. (2010). Indicadores de segurança do paciente para hospitais de pacientes agudos: revisão sistemática. Caderno de Saúde Pública, Rio de Janeiro, 26(6), 1061-1078.

Grasselli, G., Zangrillo, A., Zanella, A., Antonelli, M., Cabrini, L., Castelli, A., \& Pesenti, A. (2020). Baseline Characteristics and Outcomes of 1591 Patients Infected With SARS-CoV2 Admitted to ICU sof the Lombardy Region, Italy. Journal of the American Medical Association, 323(16), 1574-1581.

Huang, C., Wang, Y., Li, X., Ren, L., Zhao, J., Hu, Y., \& Cao, B. (2020). Clinical features of patients infected with 2019 novel coronavirus in Wuhan, China. The Lancet, 395(10223), 497-506.

Lopes, J. S. C., Jesus, P. N. L. G., Machado, T. O., \& Reis, H. F. C. (2016). Preditores de falha de extubação em unidade de terapia intensiva: uma revisão de literatura. Revista Pesquisa em Fisioterapia, 6(2), 179-188.

Loss, S. H., Oliveira, R. P., Maccari, J. G., Savi, A., Boniatti. S. A., Hetzel, M. P., \& Teixeira. S. A. (2015). A realidade dos pacientes que necessitam de ventilação mecânica prolongada: um estudo multicêntrico. Revista brasileira de terapia intensiva, 27(1)

Marinho, M. C. F., Souza, D. L. B., Bahia, B. L., \& Ferreira, F. A. A. (2020) Índices preditivos utilizados pela fisioterapia para sucesso ou insucesso no desmame ventilatório fácil e difícil. Amazon Live Journal, 2(4), 1-19.

Martinez, B. P., Andrade, F. M. D., Roncalli, A., Martins, J. A., Cavalheiro, L. V., Matte, D. L. \& Karsten, M. (2021) Comunicação oficial ASSOBRAFIR COVID-19: Intervenção na insuficiência respiratória aguda. ASSOBRAFIR Ciência.

Matic, I., Titlic, M., Dikanovic, M., Jurjevic, M., Jukic, E., \& Tonkic, A. (2007). Effects of APACHE II score on mechanical ventilation; prediction and outcome. Acta Anaesthesiologica Belgica, 58(3), 177-83.

Monteiro, M. C., Barros, L. L. D. S., \& Maia, C. D. S. F. (2016). Fatores de risco associados ao agravamento de sepse em pacientes em Unidade de Terapia Intensiva. Cadernos de Saúde Coletiva, 24(4), 388-396.

Moreira, M. F., Silva, A., \& Bassini, S. R. F. (2011). Incidência de falha e sucesso no processo de desmame da Ventilação mecânica invasiva na unidade de terapia intensiva (UTI). Revista Científica Linkania Júnior, 1(1).

Moura, J. M., Bertolli, E. S., Pereira, R. M., Frutuoso, I. S., Werneck, A. L., \& Contrin, L. M. (2017). Diagnóstico de sepse em pacientes após internação em unidade de terapia intensiva. Arquivos de Ciências da Saúde, 24(3), 55-60.

Murphy, D. M., Hanchett, M., Olmsted, R. N., Farber, M. R., Lee, T. B., Hass, J. P., \& Streed, S. A. (2012). Competency in infection prevention: A conceptual approach to guide current and future practice. American Journal of Infection Control, 40(4), 296-303.

Ñamendys-silva, S. A., Gutiérrez-Villaseñor, A., \& Romero-González, J. P. (2020). Mortalidade hospitalar em pacientes com COVID-19 ventilados mecanicamente no México. Intensive Care Medicine, 46, 2086-88.

Nascimento, M. S. M., Nunes, E. M., Medeiros, R. C., Souza, W. I. M., Sousa F ${ }^{o}$, L. F., \& Alves, E. S. R. C. (2018). Perfil epidemiológico de pacientes em unidade de terapia intensiva adulto de um hospital regional paraibano. Temas em Saúde, 18(1), 247-265.

Nassar Jro ${ }^{\circ}$ A. P., \& Park, M. (2014). Daily sedative interruption versus intermittent sedation in mechanically ventilated critically ill patients: a randomized trial. Annals of Intensive Care, 4(14).

O’dwyer, G. O., Oliveira, S. P., \& Seta, M. H. (2009). Avaliação dos serviços hospitalares de emergência do programa QualiSUS. Ciência \& Saúde Coletiva, 14(5), 1881-1890

Oliveira, A. B. F., Dias, O. M., Mello, M. M., Araújo, S., Dragosavac, D., Nucci, A., \& Falcão, A. L. E. (2010). Fatores associados à maior mortalidade e tempo de internação prolongado em uma unidade de terapia intensiva de adultos. Revista Brasileira de Terapia Intensiva, 22 (3), $250-256$.

Pauletti, M., Otaviano, M. L. P. O., Moraes, A. S. T., \& Schneider, D. S. (2017). Perfil epidemiológico dos pacientes internados em um centro de terapia intensiva. Aletheia, 50(1-2), 38-46.

Pinto, W. A. M., Rossetti, H.. B., Araújo, A., Spósito Jro, J. J., Salomão, H., Mattos, S. S., Rabelo, M. V., \& Machado, F. R. (2014). Impacto de um programa de educação continuada na qualidade assistencial oferecida pela fisioterapia em terapia intensiva. Revista Brasileira de Terapia Intensiva, 26(1), 7-12.

Prado, E. P. F. (2015). Utilização de ferramentas da qualidade e indicadores em um serviço de fisioterapia de uma unidade hospitalar (Trabalho de Conclusão de Curso). Especialização em Gestão pela Qualidade e Gerenciamento de Risco em Saúde. Atualiza Cursos. Salvador, BA, Brasil.

Prece, A., Cervantes, J., Mazur, C. S., \& Visentin, A. (2016). Perfil de pacientes em terapia intensiva: necessidade do conhecimento para organização do cuidado. Cadernos da Escola de Saúde, Curitiba, 2(16), 35-48.

Rache, B., Rocha, R., Nunes, L., Spinola, P., Malik, A. M., \& Massuda, A. (2020). Necessidades de Infraestrutura do SUS em Preparo `a COVID-19: Leitos de UTI, Respiradores e Ocupação Hospitalar. Instituto de estudos para políticas de saúde; nota técnica, (3). 
Research, Society and Development, v. 10, n. 13, e344101321365, 2021

(CC BY 4.0) | ISSN 2525-3409 | DOI: http://dx.doi.org/10.33448/rsd-v10i13.21365

Ranzani, O. T., Bastos, L. S. L., Gelli, J. G. M., Marchesi, J. F., Baião, F., Hamacher, S., \& Bozza, F. A. (2021). Caracterização das primeiras 250000 internações por COVID-19 no Brasil: uma análise retrospectiva de dados nacionais. The Lancet Respiratory Medicine.

Reis, H. F. C., Almeida, M. L. O., Silva, M. F., Moreira, J. O., \& Rocha, M.S. (2013). Associação entre o índice de respiração rápida e superficial e o sucesso da extubação em pacientes com traumatismo cranioencefálico. Revista Brasileira de Terapia Intensiva, 25(3), 212-217.

Richardson, S., Hirsch, J. S., Narasimhan, M., Crawford, J. M., McGinn, T., \& Davidson, K. W. (2020). Presenting Characteristics, Comorbidities, and Outcomes Among 5700 Patients Hospitalized With COVID-19 in the New York City Area. The Journal of the American Medical Association, 323(20), 20522059

Rotta, B. P., Silva, J. M., Fu, C., Goulardins, J. B., Pires-Neto, R. C., \& Tanaka, C. (2018). Relação entre a disponibilidade de serviços de fisioterapia e custos de UTI. Jornal Brasileiro de Pneumologia, 44(3),184-189.

Runciman, W., Hibbert, P., Thomson, R., Schaaf, T. V. D., Sherman, H., \& Lewalle, P. (2009) Towards an International Classification for Patient Safety: key concepts and terms. International Journal for Quality in Health Care, 21(1), 18-26.

Silva, R. S. (2010). Avaliação do serviço de fisioterapia municipal de Piúma/ES. Perspectivas online, 4(16).

Teich, V. D., Klajner, S., Almeida, F. A. S., Dantas, A. C. B., Laselva, C. R., Torritese, M. G., \& Cendoroglo-Neto, M. (2020). Características epidemiológicas e clínicas dos pacientes com COVID-19 no Brasil. Einstein (São Paulo), 18, 1-7.

Westyby, M. D., Klemm, A., Li, L. C., \& Jones, A. (2015). Emerging Role of Quality Indicators in Physical Therapist Practice and Health Service Delivery. Physical Therapy, 95(10)

Zhan, Q., Sun, B., Liang, L., Yan, X., Zhang, L., Yang, J, \& Wang, C. (2012). Early use of noninvasive positive pressure ventilation for acute lung injury: A multicenter randomized controlled trial. Critical Care Medicine, 40(2), 455-60. 